\title{
Percepções do enfermeiro: Processo de morte e morrer
}

\author{
Perceptions of the nurse: Processo of death and die \\ Percepciones de la enfermeira: Processo de muerte y muerte
}

Recebido: 21/03/2021 | Revisado: 26/03/2021 | Aceito: 02/04/2021 | Publicado: 12/04/2021

\author{
Alexandre Ernesto Silva \\ ORCID: https://orcid.org/0000-0001-9988-144X \\ Universidade Federal de São João Del Rei, Brasil \\ E-mail: alexandresilva@ufsj.edu.br \\ Stefãne Amorim Ribeiro \\ ORCID: https://orcid.org/0000-0001-9702-1913 \\ Faculdade Asa de Brumadinho, Brasil \\ E-mail: stefanearibeiro@yahoo.com.br \\ Gleison José Ferreira \\ ORCID: https://orcid.org/0000-0003-2961-9723 \\ Universidade Federal de São João Del Rei, Brasil \\ E-mail: gleisonenf@gmail.com \\ Jessica Maria Dalivete Silva \\ ORCID: https://orcid.org/0000-0003-3873-3093 \\ Universidade Federal de São João Del Rei, Brasil \\ E-mail: jdaliveteufsj@gmail.com \\ Luiz Alberto de Oliveira \\ ORCID: https://orcid.org/0000-0003-0682-0256 \\ Universidade Federal de São João Del Rei, Brasil \\ E-mail: albertoufsj@hotmail.com \\ Sara Batista de Jesus \\ ORCID: https://orcid.org/0000-0001-5202-7465 \\ Universidade Federal de São João Del Rei, Brasil \\ E-mail: sarabatistaenf@gmail.com \\ Thayane Vieira Carvalho \\ ORCID: https://orcid.org/0000-0001-8644-8954 \\ Clínica Auge, Brasil \\ E-mail: thayanevieira100@yahoo.com.br
}

\begin{abstract}
Resumo
Introdução: A tônica da morte e o processo de morrer inerente a ela se manifestam presentes no imaginário comum da sociedade, ocasionando inquietação e desconsolo. Para o enfermeiro a morte é compreendida como a negação de seu propósito em resguardar vidas, contudo, intensifica-seà comoção no fim da vida de uma criança ou um adulto jovem mais que a de um idoso adoecido, devida a interrupção prematura de um ciclo. Objetivo: Identificar e analisar as percepções da equipe de enfermagem no processo de morte de uma Unidade de Pronto Atendimento (UPA), frente ao processo de morrer. Método: Trata-se de um estudo qualitativo e os dados foram coletados por entrevista semiestruturada, no mês de julho de 2015. As informações extraídas das entrevistas foram analisadas pelo método de análise de conteúdo. Resultados: A equipe de Enfermagem demonstrou um enorme desconforto e inquietação com o tema morte, causando constante estresse laboral. Conclusão: Existem crenças e sentimentos negativos que margeiam o profissional de enfermagem frente à finitude dos pacientes, o que evidencia a necessidade de capacitação dos profissionais sobre esse assunto.
\end{abstract}

Palavras-chave: Atitude frente à morte; Enfermagem; Morte.

\begin{abstract}
Introduction: The tonic of death and the process of dying inherent to it are present in the common imagination of society, causing restlessness and dismay. For the nurse, death is understood as the denial of its purpose in safeguarding lives, however, the commotion intensifies at the end of the life of a child or young adult rather than a sick elderly person, due to the premature interruption of a cycle. Objective: To identify and analyze how perceptions of the nursing team in the death process of an Emergency Care Unit (UPA), in the face of the death process. Method: This is a qualitative study and data were collected through semi-structured interviews, in July 2015. The information extracted from the findings was analyzed using the content analysis method. Results: The Nursing team, a huge discomfort and uneasiness with the death theme, causing constant work stress. Conclusion: There are negative beliefs and feelings that border the nursing professional in view of the patients' finitude, which shows the need for training professionals on this subject.
\end{abstract}

Keywords: Attitude towards death; Nursing; Death. 


\begin{abstract}
Resumen
Introducción: La tónica de la muerte y el proceso de morir inherente a ella están presentes en el imaginario común de la sociedad, provocando inquietud y consternación. Para el enfermero, la muerte se entiende como la negación de su propósito de salvaguardar vidas, sin embargo, la conmoción se intensifica al final de la vida de un niño o joven adulto más que de un anciano enfermo, debido a la interrupción prematura de un ciclo. Objetivo: Identificar y analizar cómo percepciones del equipo de enfermería en el proceso de muerte de una Unidad de Atención de Emergencias (UPA), ante el proceso de muerte. Método: Se trata de un estudio cualitativo y los datos se recolectaron mediante entrevistas semiestructuradas, en julio de 2015. La información extraída de los hallazgos se analizó mediante el método de análisis de contenido. Resultados: El equipo de Enfermería, un gran malestar y malestar con el tema de la muerte, provocando un estrés laboral constante. Conclusión: Existen creencias y sentimientos negativos que bordean al profesional de enfermería ante la finitud de los pacientes, lo que evidencia la necesidad de formar profesionales en este tema.
\end{abstract}

Palabras clave: Actitud hacia la muerte; Enfermería; Muerte.

\title{
1. Introdução
}

A tônica da morte e o processo de morrer inerente a ela se manifestam presentes no imaginário comum da sociedade, ocasionando inquietação e desconsolo. A insegurança que se aloja em torno da imprevisibilidade do processo de morrer e da morte permeia o ser humano do início até o término de sua vida (Junior, Santos, Moura, Melo \& Monteiro, 2012).

Mesmo tendo em mente a certeza que um dia seremos acometidos por ela, à morte é um tema difícil de ser compreendido. Os desconfortos proporcionados por ela nos seres humanos, mesmo dentro do cotidiano profissional, promovem diferentes sentimentos demonstrados por meio da raiva ou revolta, pela tristeza e até mesmo pela depressão, pela barganha feita em segredo com o transcendente, e pela negação e isolamento como mecanismos de defesa do ego (Agra \& Albuquerque, 2008).

Remetendo a pluralidade cultural existente no mundo, o processo de morrer e morte foram encarados de diversas formas ao longo da história, expressando a diversidade de pensamentos e suas individualidades elaboradas. Com a quebra dos paradigmas criados pela sociedade, podem-se destacar as transformações relacionadas aos rituais de sepultamento e a ascensão da institucionalização de doentes e idosos, mudando o leito de morte da pessoapara dentro dos hospitais, sendo a morte vivenciada constantemente pelos profissionais da saúde, responsáveis pelos cuidados nos momentos de finitude humana (Ribeiro \& Fortes, 2012; Mota, Gomes, Coelho, Lunardi Filho \& Sousa, 2011).

Neste cenário, a enfermagem tem importante relação com as pessoas hospitalizadas em virtude da responsabilidade na promoção do bem estar e saúde envolvendo os enfermos, passando grande parte do tempo ao seu lado. Essa proximidade pode ser tanto positiva quanto negativa, em razão a frequente sobrecarga emocional consequente à morte, uma vez que os sentimentos e pensamentos decorrentes a uma precária elaboração do luto proporcionam sofrimento e angustia, produzindo uma vivência traumática, impossibilitando a transcendência desta pulsão (Dell'Acqua, Tome \& Popim, 2014; Santos \& Hormanez, 2013).

De acordo com Silva e Ruiz, para o enfermeiro a morte é compreendida como a negação de seu propósito em resguardar vidas, contudo, intensifica-seà comoção no fim da vida de uma criança ou um adulto jovem mais que a de um idoso adoecido, devida a interrupção prematura de um ciclo. Para se superar as emoções deste momento, utilizam-se da religiosidade como um importante fator de proteção, auxiliando no enfrentamento da morte, buscando nela a significação de perguntas e respostas, crendo na transcendência humana para um outro plano espiritual (Sandri, 2011; Bousso, Poles, Serafim \& Miranda, 2011).

Nesta perspectiva, as unidades de pronto atendimento (UPA) se caracterizam como ambientes de acesso irrestrito e enorme demanda de atendimentos e rotatividade, com diversidade dos quadros patológicos além de situações de carência de recursos humanos e materiais. Neste local o profissional de enfermagem está constantemente lidando com a pessoa em fase final de vida, vivenciando os temores e as frustações que envolvem essacondição, expondo o profissional a uma sobrecarga 
progressiva, proporcionando a ele um elevado nível de estresse. Assim, o tempo nesse ambiente torna-se um limitador das reflexões e de manifestações dos sentimentos relacionados ao processo de morte e morrer (Mota, Gomes, Coelho, Lunardi Filho \& Sousa, 2011; Seleghin, Mombelli, Oliveira, Waidman \& Marcon, 2012; Menzani \& Bianchi, 2009).

Mesmo que os profissionais da saúde estejam atualmente esclarecidos sobre o tema em destaque, pouco se fala em assuntos a respeito de como lidar com o processo de morrer e morte. Existem lacunas se tratando de assuntos que proporcionam análise sobre a morte que poderiambeneficiar o profissional, tanto em seu aspecto físico quanto no emocional, durante o processo de cuidar do paciente e sua familia, em situações de finitude humana. Desta maneira, reconhecer as produções imaginárias dos profissionais diante do processo de morrer, atentando para seus anseios, suas crenças, percepções e sentimentos, permite-se um aprofundamento sobre os sentimentos do profissional e o acolhimento dos pacientes e suas famílias (Martín, Riovalle \& García, 2012; Dias, Backes, Barlem, Backes, Lunardi \& Sousa, 2014; Silva, Ribeiro, Carvalho, Laia, Ferreira, \& Oliveira, 2020).

Portanto, é necessário identificar as percepções da equipe de enfermagem que trabalham em Unidades de Pronto Atendimento (UPA) no enfrentamento do processo de morte dos pacientes em seu cotidiano e propor intervenções satisfatórias para qe o processo de morte seja vivenciado da melhor forma pelo profissional de Enfermagem.

\section{Metodologia}

Trata-se de um estudo de abordagem descritiva e caráter qualitativo. A pesquisa qualitativa dedica-se a compreender os seres humanos na sua natureza própria inseridos em seu meio (Aquino, Serafim, Barbosa, Cirne, Ferreira \& Dantas, 2010). Esse tipo de pesquisa direciona-se a interpretar a experiência humana, tal como é definida e vivida pelos participantes e suas percepções. A análise dos dados da pesquisa se desenvolveu com base na descrição de conteúdo das entrevistas obtidas, tendo como referencial metodológico a análise de conteúdo (Bardin, 2011; Pereira, Fortes \& Mendes, 2013).

A pesquisa foi realizada em uma Unidade de Pronto Atendimento pública, localizada em uma cidade do centro-oeste de Minas Gerais, no mês de julho de 2015.

Foram excluídos técnicos de enfermagem e enfermeiros que atuaram em assistência a urgência e emergência há menos de 6 meses e aqueles que manifestaram desejo de não participar da pesquisa ou estiveram ausentes da unidade por motivos de licença e outros. O projeto foi aprovado pelo Comitê de Ética em Pesquisa da Universidade Federal de São João Del Rei (CEPES), conforme CAAE $\mathrm{n}^{\circ}$ 43795715.8.0000.5545. Dessa forma, ressalta-se que o estudo foi realizado segundo a Resolução 466/2012 do Conselho Nacional de Saúde (CNS) do Ministério da Saúde, que dispõe sobre diretrizes e normas regulamentares da pesquisa envolvendo seres humanos. Todos os entrevistados leram, concordaram e assinaram o Termo de Consentimento Livre e Esclarecido (TCLE).

A coleta de dados foi realizada através de uma entrevista, utilizando um instrumento elaborado pelos autores, constituído de duas partes: a primeira abordava aspectos relacionados à caracterização sócio demográfica dos sujeitos e a segunda um roteiro semiestruturado com perguntas baseadas no objetivo do estudo, com critério de saturação de informação, submetida à análise de conteúdo, está organizada em três fases, a pré-análise, a exploração do material e o tratamento dos resultados, inferência e interpretação (Bardin, 2011).

Os dados foram coletados através de entrevista com roteiro semiestruturado com as seguintes questões norteadoras: Qual o significado de morte para você? Após a morte de um paciente atendido por você, como você lida com suas emoções? Diante da possibilidade constante de morte em seu ambiente de trabalho, como institucionalmente esta situação é trabalhada?

As entrevistas foram gravadas em Mp4 para posterior transcrição. Todas as entrevistas foram codificadas com as letras ENF para enfermeiros e TEC para técnicos, seguidas de um número de ordem: ENF1... ENF12, TEC1... TEC12.

Participaram da pesquisa 17 profissionais, dos quais 10 são Enfermeiros e 7 técnicos de Enfermagem. Todos 
assinaram o termo de consentimento livre e esclarecido, onde consta o objetivo da pesquisa e a garantia do anonimato para os respondentes, de acordo com a Resolução 466/12 (Diretrizes e Normas Regulamentadoras de Pesquisas), do Conselho Nacional de Saúde (CNS)

Foi garantido o sigilo das informações, o anonimato dos participantes, bem como a possibilidade de deixar de participar deste estudo a qualquer momento, mesmo após ter assinado o termo de consentimento. O participante teve a liberdade de retirar seu consentimento, em qualquer fase da pesquisa, sem prejuízos morais ou penalizações.

Após a coleta dos dados, foi realizada a análise de conteúdo, com a pré-análise e a exploração dos dados, subdividindo os dados que emergiram em categorias, e a interpretação dos resultados articulados com a literatura pesquisada.

\section{Resultados}

As crenças espirituais e pessoais para o enfrentamento do processo de morrer, proporcionou à equipe de Enfermagem placidez e empatia ao responder às inquietações da pessoa em condição de finitude humana e de sua família, tornando-se fonte de alívio a estes.

É importante evidenciar a falta de suporte institucional relacionado ao tema proposto, visto a importância do auxílio psicológico para superar estas situações.

Com base nos relatos, foram criadas três diferentes categorias: Sentimentos vivenciados frente à morte no ambiente de trabalho; A morte sendo retratada através da crença; Suporte institucional ao profissional diante a morte.

\section{Sentimentos vivenciados frente à morte no ambiente de trabalho}

O profissional de enfermagem vivencia os últimos momentos de vida do paciente frequentemente, gerando uma sobrecarga emocional árdua e intensa, seguida de uma precária elaboração do luto, ou seja, quando não se permite a expressão da tristeza, trazendo-lhes terríveis consequências, como a maior possibilidade de adoecimento. As falas seguintes certificam esse levantamento:

Com as experiências da profissão eu vou amadurecendo e fortalecendo. (ENF9)

[...] A experiência me trouxe mecanismos para ficar de fora da situação. Trato as crianças crônicas com amor, mas vou trabalhando dentro de mim que ela não vai ficar aqui, que a morte para ela é melhor, pela patologia. (ENF4)

No princípio eu me envolvia muito sentimentalmente, mas aprendi que aquela dor, não é minha, e sim dos parentes. Então, hoje, eu separo melhor o paciente da vida pessoal, para não sofrer. (TEC6)

[...] eu penso que principalmente a gente que lida com a questão saúde doença, então a gente tem que fazer tudo aquilo bem feito pra que a gente não venha a arrepender daquilo que deixou de fazer, entendeu? Pro outro. (ENF6)

\section{A morte sendo retratada através da crença}

A crença é vista como um fator relevante para os profissionais, que creem ser Deus o acolhedor dos mortos, os recebendo de corpo e alma, proporcionando a esses profissionais um alivio momentâneo, por assim acreditarem.

Bom, pra mim a morte é claro, o fim aqui né, mas pra mim que acredito muito em Deus, eu sei que tem algo depois, então assim é o final da nossa jornada aqui, é uma coisa que a gente tem que passar por isso, mas eu acredito que tem algo depois então eu não sofro tanto assim com a morte, não. (ENF14) 
Acho que é uma passagem, eu penso que a gente ta aqui por passagem, que tem outra coisa depois da morte, eu penso em uma coisa maior depois. (ENF16)

[...] a morte não acabou é o começo. Não sou espírita, sou católico, mas eu penso dessa maneira entendeu. Ela continua, a pessoa fez uma passagem que ela ta fazendo, nesse sentido assim. (ENF19)

É eu penso que a morte, ela vem pra dar um descanso né, pro corpo humano, evitando sofrimento que a gente tem às vezes a pessoa pode estar sofrendo muito, é um descanso e de acordo com o que eu acredito, com a minha religião, a pessoa vai descansar até que deus possa trazer ela de volta a vida. (ENF17)

Então eu, por eu ter uma base evangélica muito firmada, a morte pra mim não significa final ela significa a espera de um novo começo né. É como se fosse o termino de um período que a pessoa tem na terra né, que ela vai descansar para aguardar no caso, biblicamente falando, a volta de Jesus Cristo e a gente tem que realmente estar preparada para isso, mas não considero como finitude, como final de um processo. (ENF14)

\section{Suporte institucional ao profissional diante a morte}

O profissional da saúde defronta-se com intensos dilemas existenciais frente à morte no dia a dia do trabalho. Muitas vezes, ainda como estudante, não foi instigado a pensar sobre o processo de morte e morrer, podendo ser atingido de maneira inesperada pelo pesar e não conseguir cuidar de forma adequada do indivíduo que está vivenciando a finitude da vida. Relatam que o auxílio da instituição é de grande valia para a preparação destas situações, porém, não é o que eles encontram como apoio na instituição, como se verifica nas frases seguintes:

Tem caso que marca a gente, você fica angustiada, vive o luto com a família e não temos muito a quem recorrer para ajudar nesse processo. (TEC5)

[...] eu particularmente fico muito afetado, psicologicamente eu fico abalado mesmo sabendo que é um paciente terminal, qualquer paciente que morre aqui no meu setor eu fico abalado até hoje, com 35 anos de profissão me dá um mal estar [...]. (TEC11)

Pela instituição não tem nenhum trabalho a ser feito, eu já fiz vários cursos inclusive no Einstein sobre comunicação de más notícias então eu me sinto preparada pra isso não que a instituição tenha me preparado. (ENF13)

Acho importante possuir uma equipe de psicólogos disponível, mas não somente ter psicólogos disponíveis, mas haver um trabalho em grupo com toda a equipe e a presença de outros profissionais como o psiquiatra elou enfermeiro psiquiatra. (ENF1)

\section{Discussão}

As concepções dos sentimentos vivenciados pelos profissionais da enfermagem diante do processo de morte revelam a particularidade de suas experiências. Nota-se pelo diálogo com os entrevistados que as emoções manifestadas após a morte de uma criança ou de um adulto jovem representam uma angustia e frustração com maior intensidade se comparado com um idoso, uma vez que, no ciclo da vida habitualmente a morte se encontra presente em seu último estágio.

Desta forma, ainda que o cotidiano do trabalho reflita constante proximidade com a morte, as reações e as situações distintas são ocultadas. A intensidade e o envolvimento alternam entre o vivenciar o luto com a família, ao partilhar do sofrimento e da dor, até encontrar o ponto de equilíbrio da razão e do raciocínio, passando a adotar uma postura aparentemente fria e neutra diante das adversidades (Marques, Veronez, Sanches \& Higarashi, 2013). Sendo assim, desenvolvem mecanismos 
de defesa para bloquear os sentimentos negativos proporcionados pelo convívio constante coma morte, comumente presenciada em seu ambiente de trabalho.

Essa reflexão corrobora com uma pesquisa de revisão sistemática da literatura, expondo que os conflitos que ocorrem pela morte dos seres humanos, deixam sobressaírem sentimentos diferentes que quase sempre se exemplificam pela raiva, tristeza, pela barganha e pela negação, sentimentos esses que necessitam de discussão e analise para melhor enfrentamento (Junior, Santos, Moura, Melo \& Monteiro, 2012).

Configura-se nos relatos dos entrevistados a carência de preparo em lidar com o sofrimento imputado a família e a eles mesmos, levando-os a um comportamento mecanicista comprovando-se a necessidade de uma preparação. Todavia, essa preparação deve empenhar-se no cuidado individual e psicológico, a fim de oferecer melhor suporte aos familiares e àquele que estão morrendo.

A falta de conhecimento dos profissionais de enfermagem em rotinas direcionadas para a temática em questão foi salientada por estudo de um hospital em São Paulo, no qual, expôs que dos 22 profissionais de enfermagem, 59\% disseram nunca ter realizado algum treinamento para o enfrentamento da perda e morte de pessoas próximas (Aquino, Serafim, Barbosa, Cirne, Ferreira \& Dantas, 2010). Neste mesmo tema, outros estudos de uma universidade pública confirmam os discursos através de relatos de 23 discentes do curso de Psicologia, onde questionaram que durante sua formação não é abordado assuntos vinculados ao processo de morte e morrer, o que de certa forma, causa insatisfação em alguns alunos, pela falta de compreensão e informação (Bousso, Poles, Serafim \& Miranda, 2011).

Neste estudo torna-se evidente a falta de preparo no enfrentamento da morte, por parte da instituição oferecendo parcialmente ou não oferecendo o apoio psicológico necessário para os profissionais vinculados a este processo de perda, e aos profissionais a falta de embasamento teórico e mental, fundamentais para superar este momento.

Esclarece-se ainda na elucidação dos profissionais de enfermagem a caracterização da morte como "novo começo", ou seja, imortalidade da alma após a morte, definição essa, margeada de simbologias de cunho religioso. A crença, a fé, e a religião em si tornam-se um dos alicerces, no que se refere às relações conflituosas emanadas do processo de morte, já que é frequentemente vivenciada no cotidiano de trabalho dos entrevistados.

A religião é constantemente relacionada ao processo de pós-morte, onde o milagre é aguardado sendo considerado em diversos planos. Considera-se desta forma a morte não como um processo natural e biológico, e sim como uma continuação do ser humano espiritualmente e socialmente, demonstrando as variadas representações da crença humana diante das experiências da morte (Araújo \& Belém, 2010), logo a morte não representa o fim, e sim uma transição encoberta de contemplações entre o mortal e o imortal.

De acordo com o estudo realizado de agosto de 2009 a agosto de 2011, com enfermeiros da Oncologia e Unidade de Terapia Intensiva do Recife, relata que os enfermeiros por estarem em constante contato com o paciente, procuram e devem procurar mecanismos de defesa, sendo que neste estudo também se evidencia a religiosidade como elemento primordial para o benefício do paciente e do profissional em relação a aceitação da morte, trazendo equilíbrio entre a efetividade e o distanciamento, evitando o sofrimento (Abrão, Góis, Souza, Araujo, Cartaxo \& Olveira, 2013).

A equipe, no instante em que o paciente falece normalmente busca atrelar a espiritualidade para a compreensão do fenômeno na vivência do luto. O conforto alcançado nesta circunstância pela aceitação de que esta pessoa passou para um plano superior, que seu descanso foi uma maneira de aliviar o sofrimento que a doença estava lhe proporcionando, estando melhor na companhia do Senhor, trás tranquilidade mental e espiritual para os profissionais envolvidos e para os familiares. Observa-se que crença é a única forma de enfrentamento neste instante, uma vez que, o profissional da enfermagem não recebe apoio por parte da instituição. 
De acordo com os estudos de Araújo \& Belém, as organizações hospitalares disponibilizam poucos ou nenhum apoio psicológico para as famílias e as equipes que vivenciam diariamente o momento da morte, o que acarreta sobrecarga emocional gerando agravos à saúde mental dos profissionais de enfermagem (Araújo \& Belém, 2010).

A falta de amparo profissional ocasiona uma lacuna na oferta de suporte psicológico e mental. A interdição na elaboração do luto se torna um obstáculo para saúde pública, dado o elevado número de pessoas que adoecem em função de uma excessiva carga de tristeza e sofrimento sem a possibilidade de estruturação, afetando de forma indiscriminada os mais experientes profissionais de saúde, que muitas vezes não têm espaço para velar sua própria dor.

Sendo assim, constata-se a relevância da educação permanente e da qualificação deste profissional, trazendo a ele o conhecimento por meio de capacitações e treinamentos, entendido como uma necessidade da instituição, para que possa levar a diminuição do sofrimento e da somatização advindas desse processo (Oliveira, Amaral, Viegas \& Rodrigues, 2013).

Os profissionais que buscaram apoio ou obtiveram ajuda em outras instituições em que trabalharam, consideram-se ter um preparo superior se comparado a antes de terem este suporte, acreditando ser necessário e de grande importância o acompanhamento da equipe por psicólogos ou outros profissionais da saúde mental, com discussões e treinamentos sobre o tema.

O estudo proposto ratifica a comprovação das dificuldades encontradas pelos profissionais de enfermagem para lidar com a morte em seu cotidiano de trabalho. Os relatos evidenciam as experiências anteriores, como uma área de prática, e a concepção de recursos particulares param se enfrentar e superar os desafios propostos em lidar com o a perda e o luto.

Embora as estratégias para enfrentamento criadas para bloquear suas emoções tenham efeitos consoladores para superar os obstáculos com menor oportunidade de afetar a integridade psicoemocional, apenas as vivências passadas e suas crenças não suprem a necessidade do suporte devido aos profissionais e familiares envolvidos, comprovando, desta maneira, a ausência da organização dos serviços de saúde, e a necessidade de promover um trabalho institucional voltado para o processo de morte e morrer.

\section{Considerações Finais}

Viver e morrer são antônimos que nós, seres finitos, não podemos ignorar. Aceitar a morte do outro pode parecer fácil, porém são as diferentes formas na qual a morte se apresenta, e os vínculos que temos com o paciente, que irão ditar os nossos sentimentos, refletindo nossas limitações em lidar com esse momento.

No decorrer da entrevista ficou o incomodo em falar sobre a morte e os sentimentos que permeiam esse momento, visto culturalmente como fracasso, sendo abordada por eles a falta de preparo e a necessidade de suporte relacionado ao tema, gerando uma sobrecarga emocional e barreiras psicológicas no enfrentamento do tema em questão.

Fomentar o assunto referente a morte e suas crenças nas instituições de saúde e nas universidades de forma clara e pratica, poderá ajudar os atuais e futuros profissionais de Enfermagem a estarem preparados para lidar com o final da vida do paciente, permitindo transcender os sentimentos negativos, assimilando melhor o processo de morte.

\section{Referências}

Abrão, F. M. S., Góis, A. R. S., Souza, M. S. B., Araujo, R. A., Cartaxo, C. M. B. \& Olveira, D. C.; Representações sociais de enfermeiros sobre a religiosidade ao cuidar de pacientes em processo de morte. Revista Brasileira de Enfermagem. V.66; n. 5; p.730

Agra, L. M. C. \& Albuquerque, L. H. M. (2008). Tanatologia: uma reflexão sobre a morte e o morrer. Pesquisa Psicológica.

Aquino, T. A. A., Serafim, T. D. B., Barbosa, E. L., Cirne, E. A., Ferreira, F. R. \& Dantas, P. R. S. (2010). Visões de morte, ansiedade e sentido da vida: um estudo correlacional. Psicologia Argumento. 28(63).

Araújo, S. A. N. \& Belém, K. F. (2010). O processo de morte na unidade de terapia intensiva neonatal. ConScientiae Saúde. 9(2): 290-299. 
Bardin, L. Análise de Conteúdo. 4 Ed. Lisboa (Portugal): Edições 70; 2011.

Bousso, R. S., Poles, K., Serafim, T. D. S. \& Miranda, M. G. D. (2011). Crenças religiosas, doença e morte: perspectiva da família na experiência de doença. Revescenferm USP. 45(2): 397-403.

Dell'Acqua, M. C. Q., Tome, L. Y. \& Popim, R. C. (2014). O processo de trabalho em urgência e emergência em interface com a morte. Revista da Rede de Enfermagem do Nordeste-RevRen. 14(6).

Dias M. V., Backes, D. S., Barlem, E. L. D., Backes, M. T. S., Lunardi, V. L. \& Souza, M. H. T. (2014). Formação do enfermeiro em relação ao processo de morte-morrer: percepções à luz do pensamento complexo. Revista Gaúcha de Enfermagem. 35.4: 79-85.

Júnior, S. F. J. G. S., Santos, L. C. S. S., Moura, P. V. S., Melo, B. M. S. \& Monteiro, C. F. S. (2012). Processo de morte e morrer: evidências da literatura científica de enfermagem. Revista brasileira de enfermagem. 64.6: 1122-1126.

Marques, C. D. C., Veronez, M., Sanches, M. R. \& Higarashi, I. H. (2013). Significados atribuídos pela equipe de enfermagem em unidade de terapia intensiva pediátrica ao processo de morte e morrer. Revista Mineira de Enfermagem. 17(4): 823-837.

Martín, A. M. M., Riovalle, J. S. \& García, I. G. (2012). Knowledge of the Andalusian legislation on dignified death and perception of the formation in attention to terminally ill patients of helth sciences students at Universidad de Granada, Spain. Investigación y educaciónen enfermeira. 30(2), $215-223$.

Menzani, G., \& Bianchi, E. R. F. (2009). Stress dos enfermeiros de pronto socorro dos hospitais brasileiros. Revista eletrônica de Enfermagem. 11(2), 327.

Mota, M. S., Gomes, G. C., Coelho, M. F., Lunardi Filho, W. D. \& Sousa, L. D. (2011). Reações e sentimentos de profissionais da enfermagem frente à morte dos pacientes sob seus cuidados. Revista Gaúcha Enfermagem. 32(1): 129-35.

Oliveira, P. P. D., Amaral, J. G., Viegas, S. M. D. F. \& Rodrigues, A. B. (2013). Percepção dos profissionais que atuam numa instituição de longa permanência para idosos sobre a morte e o morrer. Ciência saúde coletiva. 18(9): 2635-44.

Pereira, A. T. G., Fortes, I. F. L., \& Mendes, J. M. G. (2013). Comunicação de más notícias: revisão sistemática da literatura. Revista Enfermagem Ufpe,7(1). 227-235.DOI: 10.5205/reuol.3049-24704-1-LE.0701201331.

Ribeiro, D. B. \& Fortes, R. C. (2012). A morte e o morrer na perspectiva de estudantes de enfermagem. Revista de Divulgação Científica Sena Aires. Pag. 3239.

Sandri, C. I. M. (2011). As percepções dos enfermeiros diante da morte e do morrer em uma unidade de urgência e emergência. Anais do congresso de fenomenologia da região centro-oeste. Minas Gerais. P.135.

Santos, M. A. \& Hormanez, M. (2013). Atitude frente à morte em profissionais e estudantes de enfermagem: revisão da produção científica da última década. Revista Ciência \& Saúde Coletiva. 18(9).

Seleghim, M. R., et al. "Sintomas de estresse em trabalhadoras de enfermagem de uma unidade de pronto socorro." Revista Gaúcha de Enfermagem. 2012; 33.3: $165-173$.

Silva, A. E., Ribeiro, S. A., Carvalho, T. V., Laia, D. H. S., Ferreira, G. J. \& Oliveira, L. A. (2020). The perception of the nurse professional in front of the communication of difficult news. Research, Society and Development, [S. l.], v. 9, n. 12. DOI: 10.33448/rsd-v9i12.11014. 